\title{
Inhibition of SIRTI combined with gemcitabine therapy for pancreatic carcinoma
}

This article was published in the following Dove Press journal:

Clinical Interventions in Aging

10 July 2013

Number of times this article has been viewed

\author{
Dao-Jun Gong' \\ Jia-Min Zhang' \\ Min Yu' \\ Bo Zhuang' \\ Qing-Qu Guo² \\ 'Department of Hepatobiliary- \\ Pancreatic Surgery, Jinhua Hospital of \\ Zhejiang University, Jinhua, People's \\ Republic of China; ${ }^{2}$ Department of \\ Surgery, Second Affiliated Hospital \\ of Zhejiang University College of \\ Medicine, Hangzhou, People's Republic \\ of China
}

Background: Pancreatic carcinoma possesses one of the highest lethality rates, highest drug-resistance, and highest incidence rates. The objective of this research was to enhance the efficacy and drug-resistance for pancreatic carcinoma by using inhibition of SIRT1 combined with gemcitabine therapy methods.

Methods: Three pancreatic carcinoma cells (PANC-1 cells, BxPC-3 cells, and SW1990 cells) received treatment with physiological saline, inhibition of SIRT1, gemcitabine, and combination therapy with inhibition of SIRT1 and gemcitabine in vitro; then BxPC-3 pancreatic cancer xenogeneic mice also received treatment with physiological saline, inhibition of SIRT1, gemcitabine, and combination therapy with inhibition of SIRT1 and gemcitabine in vivo.

Results: The cleaved poly ADP ribose polymerase (PARP)-1 effect of drug in pancreatic carcinoma cells was significantly different $(P<0.05)$ and the efficacy in descending order was the combination therapy with inhibition of SIRT1 and gemcitabine, inhibition of SIRT1, and gemcitabine. The BxPC-3 pancreatic cancer xenogeneic mice model received treatment with physiological saline, inhibition of SIRT1, gemcitabine, and combination therapy with inhibition of SIRT1 and gemcitabine in vivo and the results showed that the tumor volumes decreased and the survival rate within 45 days increased according to the order of the given drugs and the difference was significant $(P<0.05)$.

Conclusion: Combination therapy with inhibition of SIRT1 and gemcitabine could improve efficacy and survival time in a BxPC-3 pancreatic cancer xenogeneic mice model, compared with single inhibition of SIRT1, or single gemcitabine therapy. The combination therapy method is a potential treatment method for pancreatic carcinoma.

Keywords: pancreatic carcinoma, combination therapy, inhibition of SIRT1-gemcitabine

\section{Introduction}

Pancreatic carcinoma is characterized with fast progression, poor prognosis, and increasing incidence. It is one of the most lethal cancers among malignant diseases with a 5 -year survival rate of about $5 \%,{ }^{1}$ and its incidence rate has increased in the past 4 decades. ${ }^{2}$ Early diagnosis of pancreatic carcinoma is still difficult, and the majority of patients are diagnosed in an advanced stage. ${ }^{2-4}$

Currently, gemcitabine is still the standard chemotherapeutic and first line drug for patients suffering from pancreatic cancer. ${ }^{5}$ However, clinical trials have confirmed that gemcitabine chemotherapy alone is not likely to achieve success because of the high intrinsic resistance of pancreatic carcinoma to currently available chemotherapy drugs. ${ }^{6-8}$ Therefore, new therapeutic methods and strategies are urgently needed.
Department of Surgery, Second Affiliated Hospital of Zhejiang University College of Medicine, 88 Jiefang Road, Hangzhou 310009 , People's Republic of China Tel +86057982318024

Fax +8605798231 8024

Email quguoqing1973@yahoo.com.cn which permits unrestricted noncommercial use, provided the original work is properly cited. 
It has been recently confirmed that SIRT1 has the antiapoptotic activity involved in tumorigenesis. ${ }^{9,10}$ In fact, higher expression of SIRT1 in a variety of cancer cell lines and tumors has been confirmed, ${ }^{11-14}$ and so we may use the inhibition of SIRT1 to promote cancer cell apoptosis and enhance therapy effects. In pancreatic carcinoma, a combination of chemotherapies seems to be the most promising strategy so far. ${ }^{15-17}$

In this study, therefore, we treated xenogeneic mice with BxPC-3 pancreatic cancer; ten were given combination therapy with inhibition of SIRT1 and gemcitabine, ten were given inhibition of SIRT1 chemotherapy, ten were given gemcitabine chemotherapy, and ten were given physiological saline. Effectiveness and safety were evaluated for comparison. We hypothesized that the inhibition of SIRT1 would induce cancer cell apoptosis, enhance therapy effects, and overcome intrinsic resistance to gemcitabine.

\section{Materials and methods}

Polyvinylidene difluoride (PVDF) was purchased from EMD Millipore (Billerica, MA, USA). Gemcitabine $\mathrm{HCl}$ was purchased from Luxuriant Bamboo Chemical Tech Co (Wuhan, People's Republic of China) and inhibition of SIRT1 (sirtinol) was purchased from Sigma-Aldrich (St Louis, MO, USA). All reagents and solvents were of analytical grade or better. BxPC-3, SW 1990, and PANC-1 cells were from American Type Culture Collection (ATCC) (Manassas, VA, USA). Cells were maintained in Dulbecco's Modified Eagle Medium (DMEM) with 100 units $/ \mathrm{mL}$ penicillin, $100 \mathrm{mg} / \mathrm{mL}$ streptomycin, and $10 \%$ fetal bovine serum at $37^{\circ} \mathrm{C}$ in a humidified $95 \%$ air and $5 \% \mathrm{CO}_{2}$ atmosphere. The cells were treated with physiological saline, gemcitabine, sirtinol, and a combination of gemcitabine and sirtinol.

\section{Cell growth and death assays}

Cells were plated at 5000 cells $/ \mathrm{cm}^{2}$ and, after 24 hours, fed with fresh medium and treated with different doses of gemcitabine, sirtinol, and the combination of gemcitabine and sirtinol in complete medium. After 24, 48, and 72 hours of these treatments, total cells were collected by brief trypsinization, and washed with phosphate-buffered saline (PBS). Total cell number was determined by counting each sample in duplicate using a hemocytometer under an inverted phase contrast microscope (Olympus Corporation, Tokyo, Japan) using Trypan blue dye. Dead cells could not be excluded from the dye and showed blue staining of the cell. Each treatment at each time point was done in triplicate.

\section{Western blot and protein extraction}

The effect on the molecular level of apoptosis was observed using Western blot analysis. ${ }^{18}$ We cultured $1 \times 10^{6}$ cells $/ \mathrm{mL}$ of BxPC-3, PANC-1, and SW1990 cells for 12 hours at $37^{\circ} \mathrm{C}$ in three culture flasks of $25 \mathrm{~cm}^{2}$ (Corning Inc, Corning, NY, USA) including $10 \mathrm{~mL}$ of culture media. After 24 hours, $5 \mathrm{~mL}$ of media including physiological saline, gemcitabine, sirtinol, or the combination of gemcitabine and sirtinol in the concentration of $1 \mathrm{mmol} / \mathrm{L}$ were added to the three cell flasks and incubated for 24 hours and 72 hours at $37^{\circ} \mathrm{C}$. The three cells with physiological saline were used as a control group for the test. After the time of incubation, extracts of cells were taken by cell scraping and were washed twice with ice-cold PBS $(0.01 \mathrm{M}$, $\mathrm{pH}$ 7.4). All lysates of cells were fractionated in $50 \mathrm{~mol} / \mathrm{mL}$ Tris hydrochloride (Tris-HCl) ( $\mathrm{pH} 7.5$ ), 1\% NP40, 0.1\% sodium dodecyl sulfate (SDS), $150 \mathrm{~mol} / \mathrm{mL}$ sodium chloride $(\mathrm{NaCl}), 0.5 \%$ sodium deoxycholate containing a phosphatase inhibitor, and protease (Sigma-Aldrich) cocktails for lysing the cell extracts. The concentration of protein was determined using a micro bicinchoninic acid (BCA) assay. Cell lysates with identical protein amounts were prepared and diluted in $5 \times$ loading buffer $(0.2 \%$ bromophenol blue, 10\% glycerol, 4\% 2-mercaptoethanol, $0.32 \mathrm{~mol} / \mathrm{L}$ Tris-HCl, 2\% SDS [pH 6.8]) before loading onto $10 \%$ SDS-polyacrylamide gel.

Protein immunoassay was determined using electrophoretic transfer of SDS-polyacrylamide-gel-electrophoresis (PAGE), separating proteins to the PVDF membrane (GE Healthcare UK Ltd, Little Chalfont, UK). The PVDF was incubated in blocking buffer (Tris buffer saline [TBS] containing 5\% nonfat milk and $0.1 \%$ Tween 20 ) for 1 hour at $25^{\circ} \mathrm{C}$. Then the PVDF membrane was incubated with the appropriate primary antibody from Santa Cruz Biotechnology (Santa Cruz, CA, USA) recognizing poly ADP ribose polymerase (PARP) (in 1:10,000 dilution), Glyceraldehyde 3-phosphate dehydrogenase (GAPDH) antibody, (AP0063; Bioworld Technology, Inc., St Louis Park, MN, USA) (in $1: 10,000$ dilution), and $b$-actin (in 1:10,000 dilution) for 12 hours at $4{ }^{\circ} \mathrm{C}$ or for 1 hour at $25^{\circ} \mathrm{C}$ with shaking. The PVDF membrane was washed three times with TBS (containing $0.1 \%$ Tween 20 ) for 10 minutes and then incubated with goat anti-mouse/rabbit immunoglobulin $\mathrm{G}$ (IgG)-horseradish peroxidase conjugated secondary antibody (Santa Cruz Biotechnology) for 1 hour at $25^{\circ} \mathrm{C}$. After repeating the wash processes three times, the target protein was incubated with Enhanced Chemiluminescence Plus (Amersham Biosciences, Little Chalfont, UK) and then exposed to film. 


\section{Tumor xenogeneic study}

To obtain BxPC-3 tumor mice, BxPC-3 cells were cultured and proliferated, then detected by trypsinization, washed, and resuspended in serum-free DMEM. Six-week-old athymic nu/ nu male mice were subcutaneously injected with $3 \times 10^{6}$ cells to initiate tumor growth. When the volume of tumors reached $180-200 \mathrm{~mm}^{3}$, the mice were randomly divided into four groups for the study, with ten mice in each group. At day 8, 12, $16,20,24,28,32$, and 36, physiological saline, gemcitabine (dissolved in saline, $10 \mathrm{mg} / \mathrm{kg}$ equivalent to gemcitabine), sirtinol (dissolved in saline, $10 \mathrm{mg} / \mathrm{kg}$ equivalent to sirtinol), or the combination of gemcitabine (dissolved in saline, $10 \mathrm{mg} / \mathrm{kg}$ equivalent to gemcitabine) and sirtinol (dissolved in saline, $25 \mathrm{mg} / \mathrm{kg}$ equivalent to sirtinol) were given to mice via tail vein. The tumor volumes of mice were then detected using a caliper after 45 days. Tumor size was detected across its two perpendicular diameters, and its volume was calculated by the formula: volume $=\left(\right.$ long diameter $\times$ short diameter $\left.{ }^{2}\right) / 2\left(\mathrm{~mm}^{3}\right) .{ }^{19}$

At the end of the test, tumor tissues were excised (day 45), embedded in optimal cutting temperature (OCT) compound (Tissue-Tek $^{\circledR}$; Sakura Finetek USA, Inc, Torrance, CA, USA), and cryosectioned into a $7 \mu \mathrm{m}$ length using a Leica CM3050S cryostat (Leica Microsystems, Wetzlar, Germany) for immunohistological and histological analysis.

\section{Statistical analysis}

All statistical tests were two-sided and performed in SPSS version 16.0 (IBM Corporation, Armonk, NY, USA) for Windows. Curative effectiveness was compared by chisquare test, survival analysis was made by Kaplan-Meier methods, and survival rate curves were compared by the Log-Rank test.

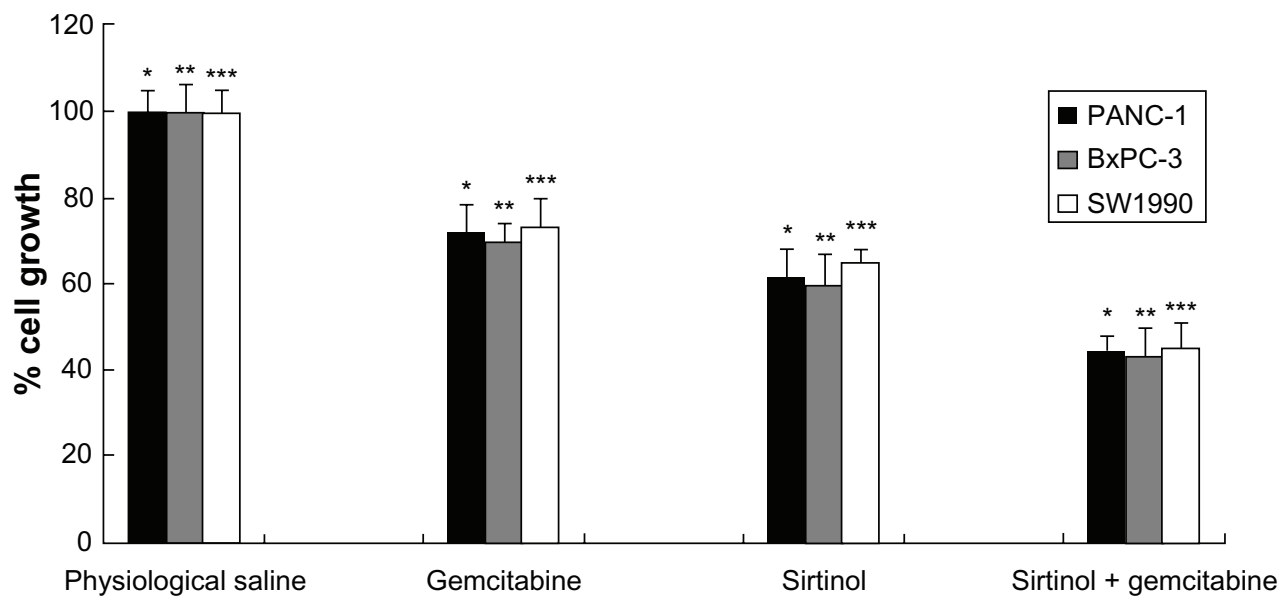

Figure I In vitro antiproliferative effect of the combination treatment of gemcitabine and sirtinol in PANC-I, BxPC-3, and SWI990 cells. Notes: $* P<0.05$, $* * P<0.05$, and $* * * P<0.05$ against physiological saline, gemcitabine, and sirtinol control. 


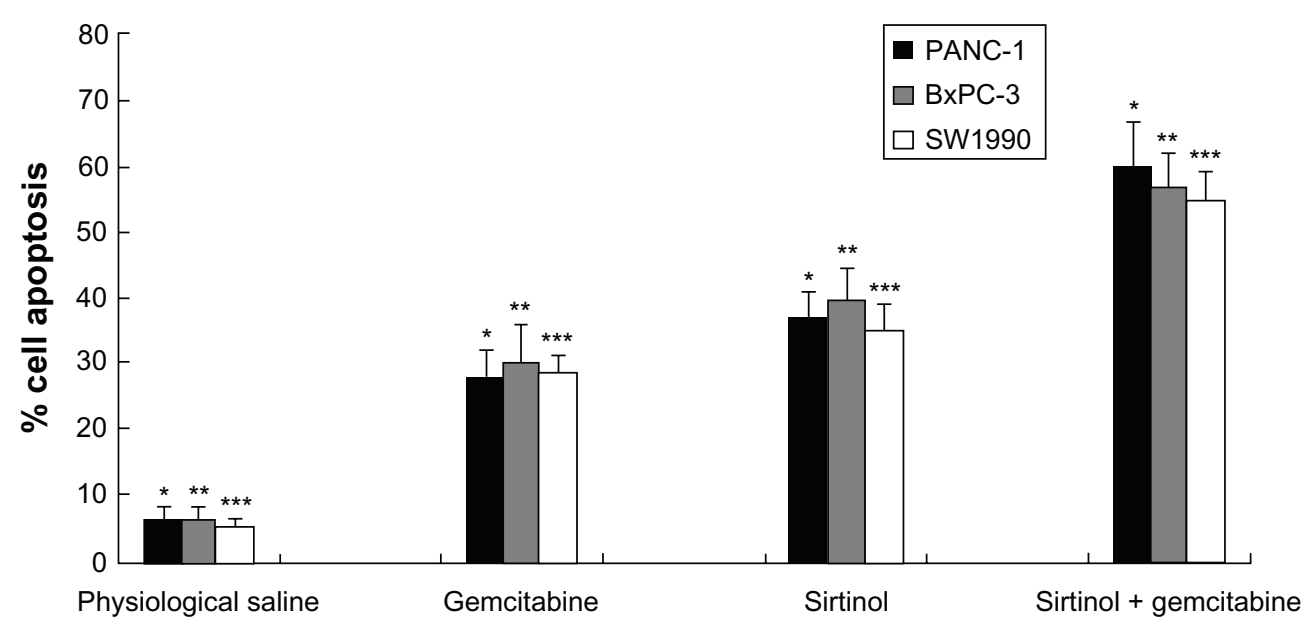

Figure 2 In vitro apoptosis effect of combination treatments of gemcitabine and sirtinol in PANC-I, BxPC-3, and SWI990 cells. Notes: $* P<0.05, * * P<0.05$, and $* * * P<0.05$ against physiological saline, gemcitabine, and sirtinol control.

This is possibly because gemcitabine alone easily induced drug resistance and sirtinol inhibited SIRT1 and induced cell apoptosis.

\section{Western blot analysis}

To confirm the result, the effect of the combination of gemcitabine and sirtinol in comparison to physiological saline, gemcitabine, or sirtinol was studied on the molecular level using Western blotting of cleaved PARP-1 antibody and PARP. PARP is a zinc-dependent DNA binding protein that recognizes DNA strand breaks and is presumed to play a role in DNA repair. ${ }^{20}$ As a marker for apoptosis, PARP is cleaved in vitro by many caspases, and in vivo by caspase- $3 .{ }^{21}$ Existing as a $116 \mathrm{kDa}$ nuclear protein, PARP is cleaved between the amino acids Asp214 and Gly215 to yield two fragments of $29 \mathrm{kDa}$ (C-terminal catalytic domain) and $85 \mathrm{kDa}$ (N-terminal DNA-binding domain). ${ }^{22,23}$

The principle was that, as control cells receiving DNA damage, a little cleaved PARP-1 would be detected because of the cell cycle arrest and thus, the cells had enough time to repair the double-strand breaks. Therefore, we detected the proteolytic cleavage of PARP, which synthesized PARP from $\beta$-nicotinamide adenine dinucleotide in response to DNA strand breaks, an early biochemical event during apoptosis. We performed a Western blot of PARP cleavage in BxPC3, PANC-1, and SW1990 cells following the combination of gemcitabine and sirtinol in comparison to physiological saline, gemcitabine, or sirtinol treatment with cleaved PARP antibodies that detect cleaved PARP ( $84 \mathrm{kDa}$ and $29 \mathrm{kDa}$ ) and intact PARP $(116 \mathrm{kDa})$. As shown in Figures 3-5, the three BxPC-3, SW1990, and PANC-1 cells treated with
$1 \mathrm{mmol} / \mathrm{L}$ of the combination of gemcitabine and sirtinol for 1 day indicated PARP cleavage to a greater extent than treatment with sirtinol, gemcitabine, or physiological saline; this confirmed that apoptosis induced by the combination of gemcitabine and sirtinol is activated more efficiently than by sirtinol, gemcitabine, or physiological saline. Hence, we think that the combination of gemcitabine and sirtinol not only enhances therapy efficacy, but also actually enhances the synergistic biological action of sirtinol and gemcitabine.

\section{In vivo antitumor effect in xenogeneic mice}

In vivo antitumor activity of the combination of gemcitabine and sirtinol was measured with a xenogeneic nude mouse model with human BxPC-3 pancreatic cancer cells. One week after the pancreatic cancer cells were subcutaneously injected in the flank of BALB/c-nu nude mice (day 7), the mice were randomly divided into four treatment groups $(\mathrm{n}=10)$. Various drugs were administered intravenously twice a week for a total of eight times: control (physiological saline), a combination of gemcitabine and sirtinol $(10 \mathrm{mg} / \mathrm{kg})$, gemcitabine $(10 \mathrm{mg} / \mathrm{kg})$, sirtinol $(10 \mathrm{mg} / \mathrm{kg})$. Tumor volume was detected on day 45 . Mouse weight loss was not significant in any of the test groups, showing that all of the treatments were well-tolerated. Tumor growth was inhibited rapidly when the mice were treated with the combination of gemcitabine and sirtinol $(10 \mathrm{mg} / \mathrm{kg})$, gemcitabine $(10 \mathrm{mg} / \mathrm{kg})$, sirtinol, and physiological saline, $(10 \mathrm{mg} / \mathrm{kg})$. There were significant differences between these four groups (Figure 6, $P<0.05$ ). After 45 days, the tumor growth inhibition by the combination of gemcitabine and sirtinol treatment was 3.0-fold higher than that by the physiological saline, 


\section{Physiological saline Gemcitabine Sirtinol Sirtinol + gemcitabine}

\section{Cleaved PARP-1}

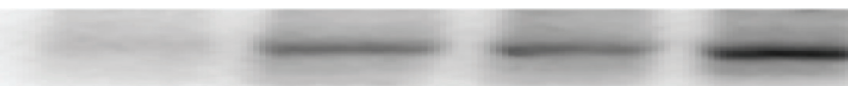

GAPDH

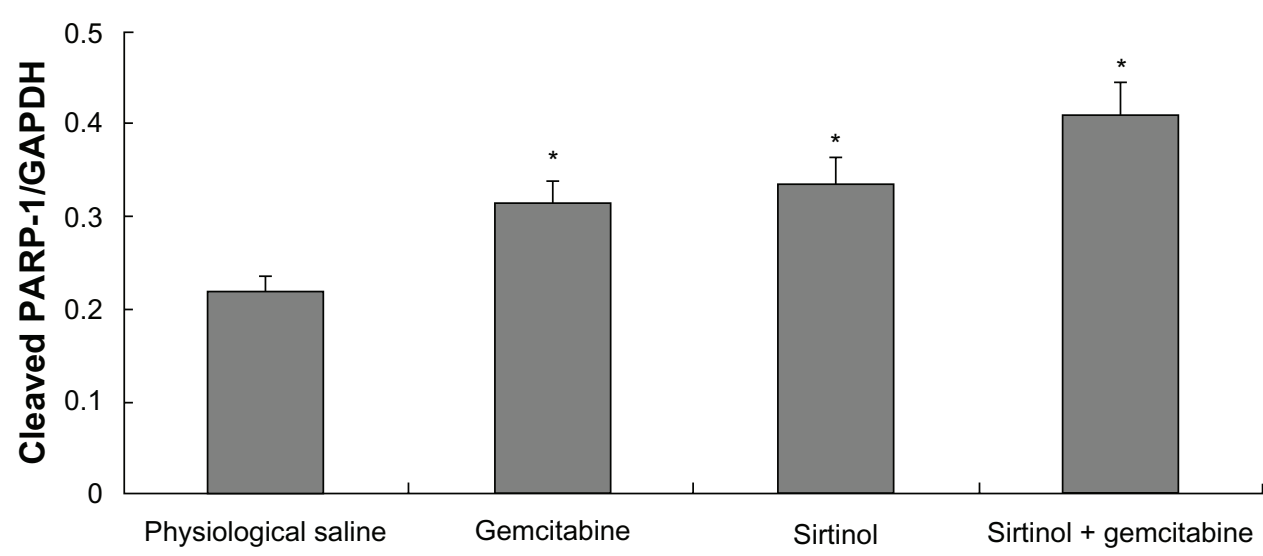

PANC-1 cells

Figure 3 In vitro cleaved PARP-I effect of drug in PANC-I cells.

Note: $* P<0.05$ against physiological saline control.

Abbreviations: GAPDH, glyceraldehyde 3-phosphate dehydrogenase; PARP, poly ADP ribose polymerase.

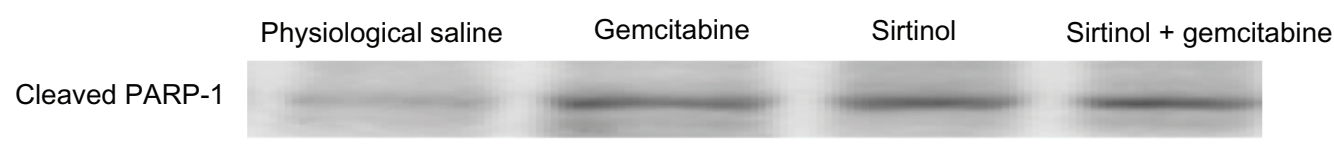

GAPDH

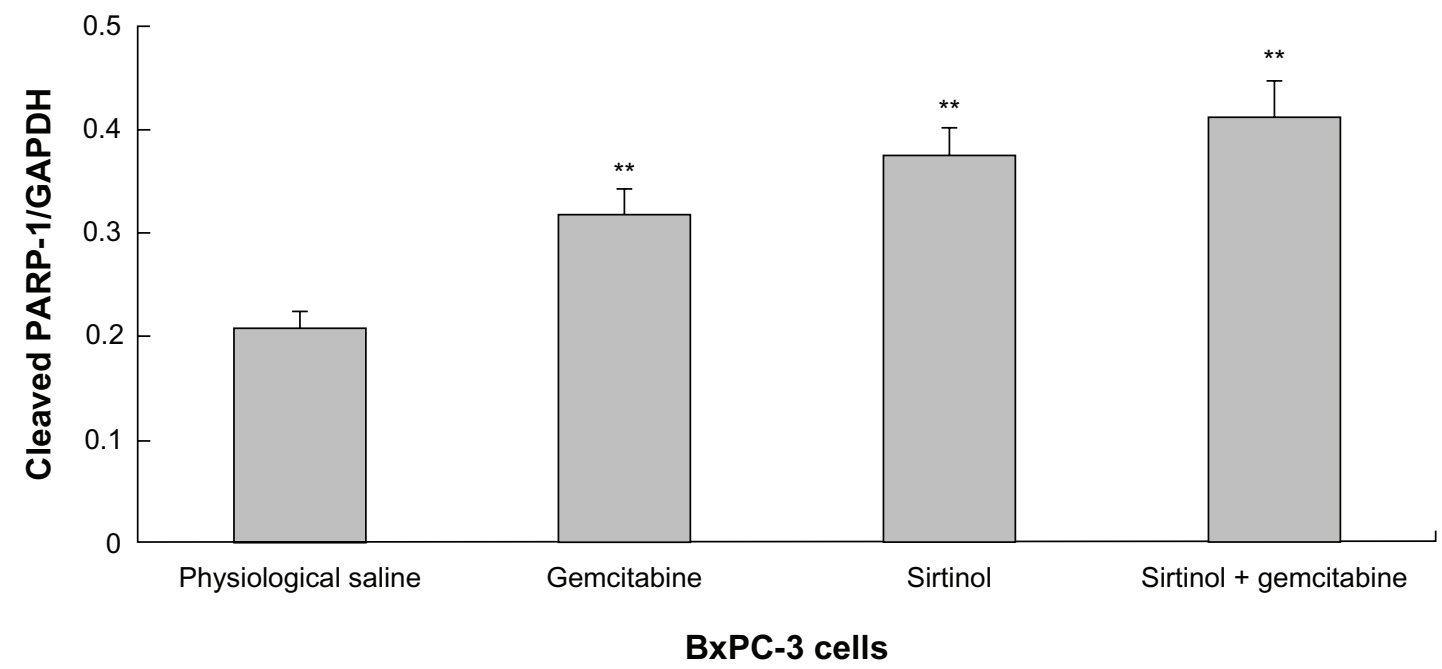

Figure 4 In vitro cleaved PARP-I effect of drug in BxPC-3 cells.

Note: $* * P<0.05$ against physiological saline control.

Abbreviations: GAPDH, glyceraldehyde 3-phosphate dehydrogenase; PARP, poly ADP ribose polymerase. 

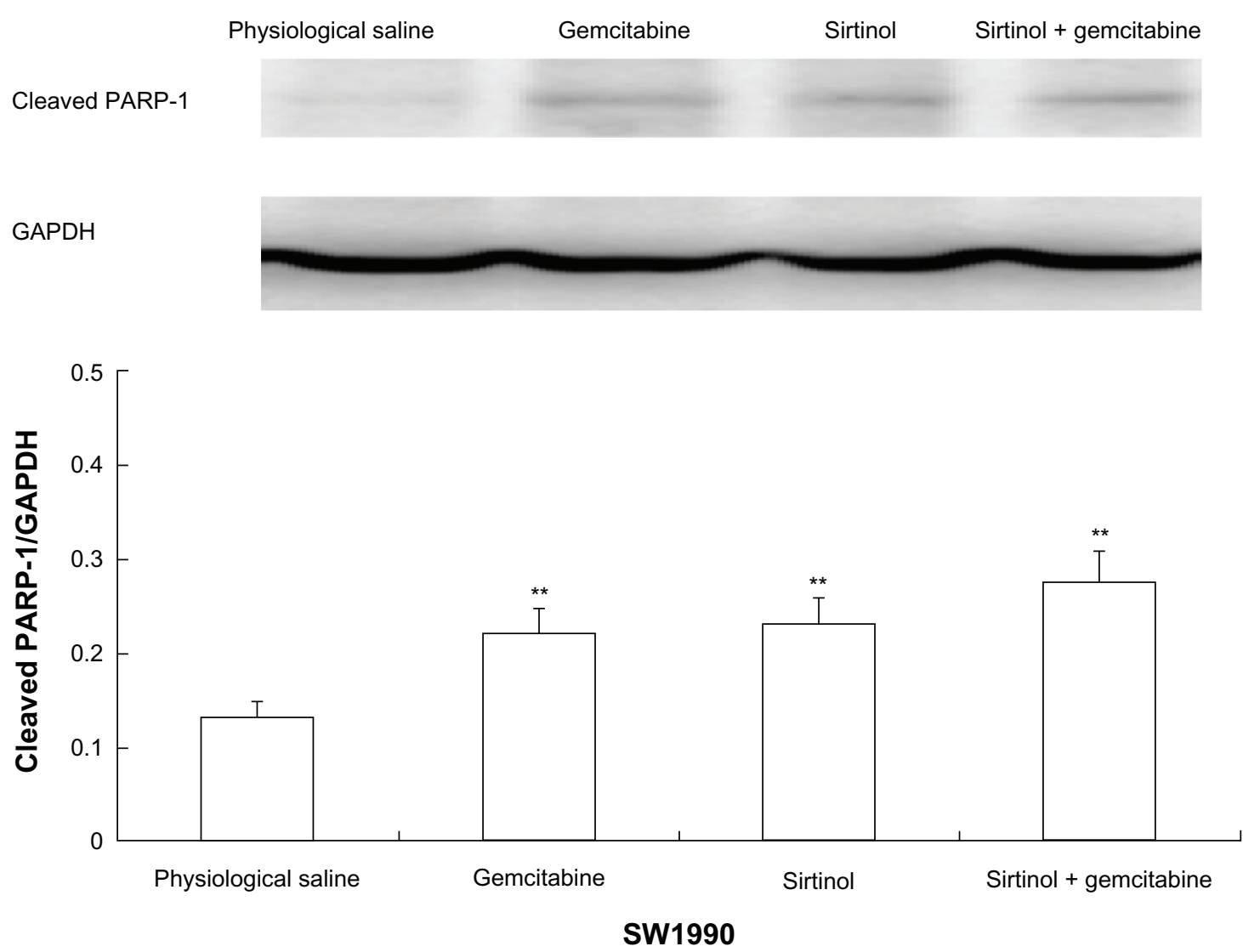

Figure 5 In vitro cleaved PARP-I effect of drug in SWI990 cells.

Note: **P $<0.05$ against physiological saline control.

Abbreviations: GAPDH, glyceraldehyde 3-phosphate dehydrogenase; PARP, poly ADP ribose polymerase.

2.5 -fold higher than that by the gemcitabine, and 1.8-fold higher than that by the sirtinol (Figure 6A-D, $P<0.05$ ). The survival ratio after 45 days of the group treated with the combination of gemcitabine and sirtinol was better than other three groups (Figure 7). This showed that the combination therapy of gemcitabine and sirtinol was effective in treating the pancreatic cancer.

\section{Tumor tissues immunohistological and histological analysis}

To investigate the effect of the combination of gemcitabine and sirtinol on the pancreatic cancer in xenogeneic nude mice, immunohistochemical staining was done with a hematoxylin and eosin (HE) assay. Figure 8 shows images of the tumor tissues taken from the xenogeneic mice 45 days after intravenous injection of physiological saline, gemcitabine, sirtinol, or the combination of gemcitabine and sirtinol. HE staining (Figure 8A-D) showed the cells in the tumor tissue site with physiological saline, gemcitabine, sirtinol, or the combination of gemcitabine and sirtinol treatment. The cells in the tumor tissue site with the combination of gemcitabine and sirtinol treatment were the nearest to normal pancreatic tissue of four treatment groups. This suggests that the combination of gemcitabine and sirtinol treatment was a promising therapy method for pancreatic cancer.

Tumors are an important cause of adult deaths worldwide. ${ }^{23}$ Unfortunately, the therapies for pancreatic cancer, including chemotherapy, radiation, and surgery, have very high risks. ${ }^{24-26}$ In recent years, combination treatments of different drugs have been developed in the field. However, combination treatments of gemcitabine and sirtinol have not been previously reported. Because sirtinol inhibits SIRT1 and could induce apoptosis in cancer cells and gemcitabine was a first-line therapy of pancreatic cancer (but drug resistance by cancer cells is common), we utilized the combination treatment of gemcitabine and sirtinol to try to overcome these barriers. The significant findings in the present study were that the combination treatment of gemcitabine and sirtinol inhibits growth of pancreatic cancer in vitro in cell cultures as well as in vivo in xenogeneic athymic nude mice. ${ }^{27}$ 

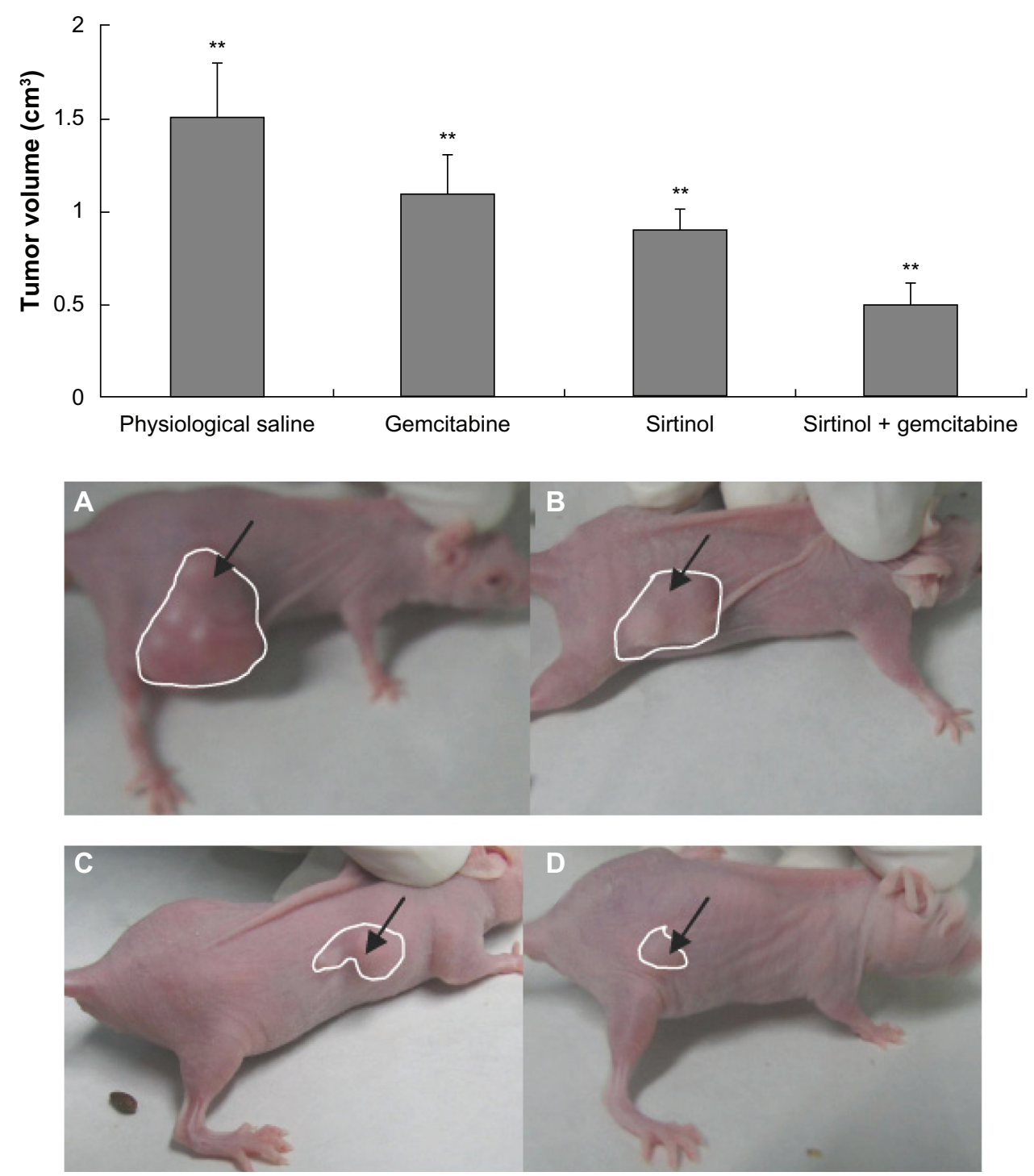

Figure 6 Tumor volume effect of different drug therapy.

Notes: (A) Physiological saline. (B) Gemcitabine. (C) Sirtinol (inhibition of SIRTI). (D) Gemcitabine and sirtinol (inhibition of SIRTI). **P < 0.05; calculated by one-way analysis of variance test. Error bars represent the standard deviation for ten mice per group.

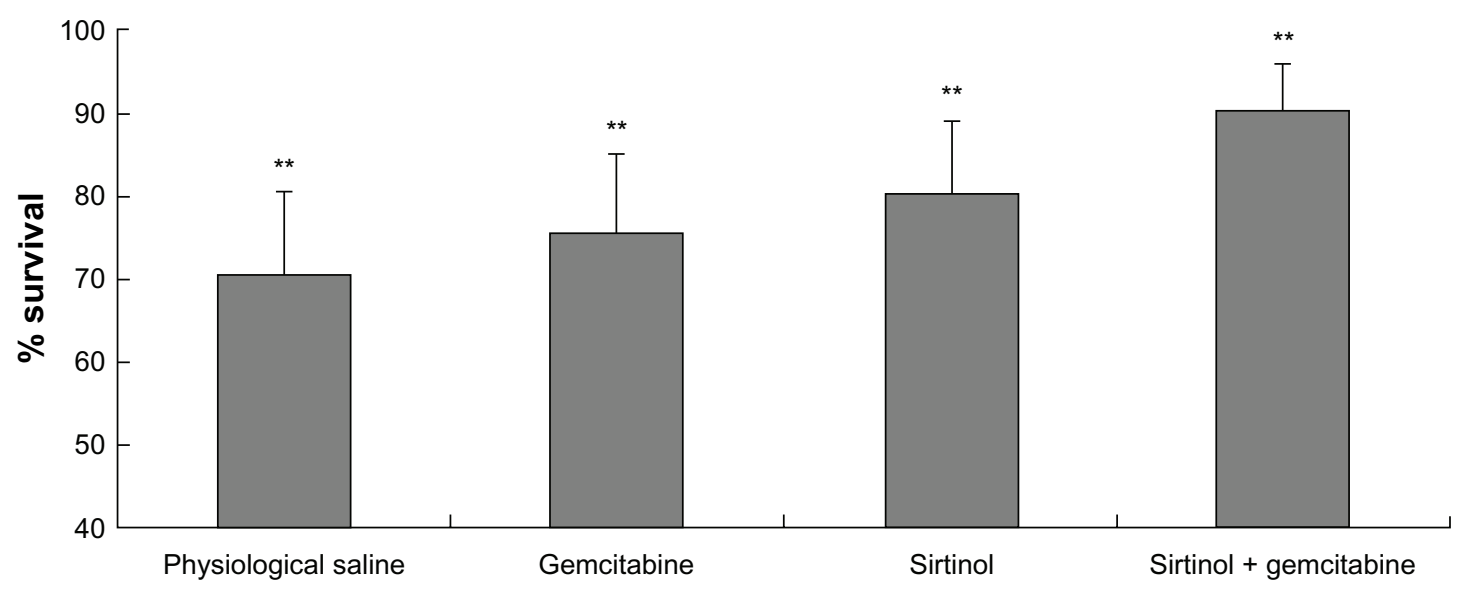

Figure 7 Improved therapeutic efficacy of sirtinol combined with gemcitabine in BxPC-3 pancreatic cancer xenograft mice for 45 days.

Note: $* * P<0.05$. 


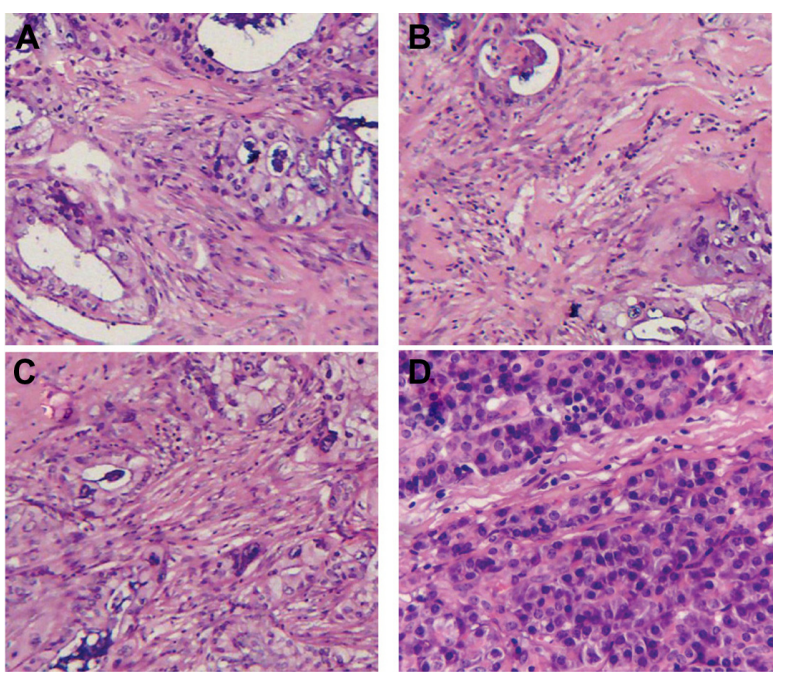

Figure 8 Hematoxylin and eosin assays of tumor tissues of the xenograft mice after various treatments.

Notes: (A) Physiological saline. (B) Gemcitabine. (C) Sirtinol (inhibition of SIRTI). (D) Gemcitabine and sirtinol (inhibition of SIRTI).

\section{Conclusion}

We proposed and designed a combination treatment of gemcitabine and sirtinol for pancreatic cancer. The combination treatment method produced a synergistic effect that enhanced the treatment effects of both gemcitabine and sirtinol. The in vivo and in vitro results showed that the method has promise for pancreatic cancer therapy in the future.

\section{Acknowledgments}

The study was supported by grants from the Foundation of the Health Department of Zhejiang Province, People's Republic of China (No 2010SSA008), the Foundation of Science and Technology Department of Jinhua (No 2010-3-003) and National Natural Science Foundation (No 81071960).

\section{Disclosure}

The authors report no conflicts of interest in this work.

\section{References}

1. Li D, Xie K, Wolff R, Abbruzzese JL. Pancreatic cancer. Lancet. 2004;363(9414):1049-1057.

2. Zhou J, Song SD, Li DC, Zhou J, Zhu DM, Zheng SY. Clinical significance of expression and amplification of the DcR3 gene in pancreatic carcinoma. Asian Pac J Cancer Prev. 2012;13(2):719-724.

3. Ikeda S, Maeshirok K, Ryu S, et al. Diagnosis of small pancreatic cancer by endoscopic balloon-catheter spot pancreatography: an analysis of 29 patients. Pancreas. 2009;38(4):102-113.

4. Klauss M, Stiller W, Fritz F, et al. Computed tomography perfusion analysis of pancreatic carcinoma. J Comput Assist Tomogr. 2012;36(2): 237-242.

5. Burris HA 3rd, Moore MJ, Andersen J, et al. Improvements in survival and clinical benefit with gemcitabine as first-line therapy for patients with advanced pancreas cancer: A randomized trial. J Clin Oncol. 1997;15(6): 2403-2413.
6. Burris H, Rocha-Lima C. New therapeutic directions for advanced pancreatic cancer: targeting the epidermal growth factor and vascular endothelial growth factor pathways. Oncologist. 2008;13(3): 289-298.

7. O'Reilly EM, Abou-Alfa GK. Cytotoxic therapy for advanced pancreatic adenocarcinoma. Semin Oncol. 2007;34(4):347-353.

8. von Wichert G, Seufferlein T, Adler G. Palliative treatment of pancreatic cancer. J Dig Dis. 2008;9(1):1-7.

9. Glozak MA, Seto E. Histone deacetylases and cancer. Oncogene. 2007;26(37):5420-5432.

10. Saunders LR, Verdin E. Sirtuins: critical regulators at the crossroads between cancer and aging. Oncogene. 2007;26(37):5489-5504.

11. Hida Y, Kubo Y, Murao K, Arase S. Strong expression of a longevityrelated protein, SIRT1, in Bowen's disease. Arch Dermatol Res. 2007; 299(2):103-106.

12. Huffman DM, Grizzle WE, Bamman MM, et al. SIRT1 is significantly elevated in mouse and human prostate cancer. Cancer Res. 2007;67(14): 6612-6618

13. Kuzmichev A, Margueron R, Vaquero A, et al. Composition and histone substrates of polycomb repressive group complexes change during cellular differentiation. Proc Natl Acad Sci US A. 2005;102(6): 1859-1864.

14. Yeung F, Hoberg JE, Ramsey CS, et al. Modulation of NF-kappaBdependent transcription and cell survival by the SIRT1 deacetylase. EMBO J. 2004;23(12):2369-2380.

15. Danovi SA, Wong HH, Lemoine NR. Targeted therapies for pancreatic cancer. Br Med Bull. 2008;87:97-130.

16. Kleespies A, Jauch KW, Bruns CJ. Tyrosine kinase inhibitors and gemcitabine: new treatment options in pancreatic cancer? Drug Resist Updat. 2006;9(1-2):1-18.

17. Ko AH. Future strategies for targeted therapies and tailored patient management in pancreatic cancer. Semin Oncol. 2007;34(4): 354-364.

18. Parsels LA, Morgan MA, Tanska DM, et al. Gemcitabine sensitization by checkpoint kinase 1 inhibition correlates with inhibition of a Rad51 DNA damage response in pancreatic cancer cells. Mol Cancer Ther. 2009;8(1):45-54.

19. VanWeelden K, Flanagan L, Binderup L, Tenniswood M, Welsh J. Apoptotic regression of $\mathrm{MCF}-7$ xenografts in nude mice treated with the vitamin D3 analog, EB1089. Endocrinology. 1998;139(4): 2102-2110.

20. Ikejima M, Noguchi S, Yamashita R, et al. The zinc fingers of human poly(ADP-ribose) polymerase are differentially required for the recognition of DNA breaks and nicks and the consequent enzyme activation. Other structures recognize intact DNA. J Biol Chem. 1990;265(35): 21907-21913.

21. Kaufmann SH, Desnoyers S, Ottaviano Y, Davidson NE, Poirier GG. Specific proteolytic cleavage of poly(ADP-ribose) polymerase: An early marker of chemotherapy-induced apoptosis. Cancer Res. 1993;53(17): 3976-3985.

22. Lazebnik YA, Kaufmann SH, Desnoyers S, Poirier GG, Earnshaw WC. Cleavage of poly(ADP-ribose) polymerase by a proteinase with properties like ICE. Nature. 1994;371(6495):346-347.

23. Tewari M, Quan LT, O'Rourke K, et al. Yama/CPP32 beta, a mammalian homolog of CED-3, is a CrmA-inhibitable protease that cleaves the death substrate poly(ADP-ribose) polymerase. Cell. 1995;81(5): 801-819.

24. Yokoyama Y, Nimura Y, Nagino M. Advances in the treatment of pancreatic cancer: limitations of surgery and evaluation of new therapeutic strategies. Surg Today. 2009;39(6):466-475.

25. Pliarchopoulou K, Pectasides D. Pancreatic cancer: current and future treatment strategies. Cancer Treat Rev. 2009;35(5):431-436.

26. Torchilin VP. Recent advances with liposomes as pharmaceutical carriers. Nat Rev Drug Discov. 2005;4(2):145-160.

27. Kalle AM, Mallika A, Badiger J, Alinakhi, Talukdar P, Sachchidanand. Inhibition of SIRT1 by a small molecule induces apoptosis in breast cancer cells. Biochem Biophys Res Commun. 2010;401(1):13-19. 
Clinical Interventions in Aging

\section{Publish your work in this journal}

Clinical Interventions in Aging is an international, peer-reviewed journal focusing on evidence-based reports on the value or lack thereof of treatments intended to prevent or delay the onset of maladaptive correlates of aging in human beings. This journal is indexed on PubMed Central, MedLine, the American Chemical Society's 'Chemical Abstracts

Service' (CAS), Scopus and the Elsevier Bibliographic databases. The manuscript management system is completely online and includes a very quick and fair peer-review system, which is all easy to use. Visit http://www.dovepress.com/testimonials.php to read real quotes from published authors.

Submit your manuscript here: http://www.dovepress.com/clinical-interventions-in-aging-journal 